\title{
Fine structure of coupled optical modes in photonic molecules
}

\author{
Y. P. Rakovich, ${ }^{1}$ J. F. Donegan, ${ }^{1}$ M. Gerlach, ${ }^{1}$ A. L. Bradley, ${ }^{1}$ T. M. Connolly, ${ }^{2}$ J. J. Boland, ${ }^{2}$ N. Gaponik ${ }^{3}$ and A. Rogach ${ }^{4}$ \\ ${ }^{1}$ Semiconductor Photonics Group, Department of Physics, Trinity College, Dublin 2, Ireland \\ ${ }^{2}$ Department of Chemistry, Trinity College, Dublin 2, Ireland \\ ${ }^{3}$ Institute of Physical Chemistry, University of Hamburg, 20146 Hamburg, Germany \\ ${ }^{4}$ Photonics and Optoelectronics Group, Physics Department and CeNS, Ludwig-Maximilians Universität München, 80799 \\ Munich, Germany
}

(Received 16 April 2004; published 8 November 2004)

\begin{abstract}
We report on the coherent coupling of whispering gallery modes (WGM) in a photonic molecule formed from two melamine-formaldehyde spherical microcavities with a thin shell of CdTe nanocrystals. Utilizing a microporous polymer structure to orient the photonic molecule, we have excited the photonic molecule both on and off axis. This controllable geometry has allowed the observation of an off-axis fine structure that consists of very sharp peaks resulting from the removal of the WGM degeneracy with respect to the azimuthal quantum number $m$. The mode splittings are in very good agreement with theory.
\end{abstract}

DOI: 10.1103/PhysRevA.70.051801 PACS number(s): 42.60.Da, 42.70.Qs, 61.46.+w, 78.67. $-\mathrm{n}$

In recent years, the modification of electromagnetic modes in solid spherical microcavities has been of great interest both for the studies of fundamental optical properties and for the potential applications [1,2]. Extending the ideas of the linear combination of atomic orbitals method to the classical wave case, it was shown that Mie resonances of single microspheres play the same role as the atomic orbitals in the electronic case and the spatial distributions of WGM can be described by analogy with the orbitals in a hydrogen atom [3]. Following the analogy with quantum mechanics, three integers, $n, l$, and $m$, describing whispering gallery modes (WGM), correspond to angular, radial and the azimuthal quantum numbers, respectively. This approach has enabled small dielectric spheres to be considered as "photonic atoms" $[3,4]$.

The resonant internal field of the microsphere is not fully confined and an evanescent field extends into the surroundings. It was recently recognized that this evanescent field would allow for coherent coupling between WGM of two adjacent spherical particles with closely matched sizes. Such a system of coherently coupled "photonic atoms" may be called a "photonic molecule" (PM) [5] and can be employed in order to control the density of photon states in the micrometer length scale. In analogy to the formation of molecular electronic orbits, the tight binding approximation provides two combinations for the electromagnetic field in a system of interacting microspheres: bonding $(\mathrm{BN})$ and antibonding $(\mathrm{ABN})$ states [5-8]. Experimentally, the coupling of the photon modes of individual microspheres in the PM can cause a narrow resonance of a photonic atom to split into two modes of lower quality factor [6]. This phenomenon has been clearly demonstrated in a system of two square, photonic dots coupled by a narrow channel [5], in a dye-stained bisphere system $[7,9,10]$ and in chains of polymer-blend microparticles [11]. Detailed theoretical considerations of coherent mode coupling, however, predicts a more complex internal distribution of density of photonic states within $\mathrm{BN}$ and $\mathrm{ABN}$ modes [8].

In this paper we report on the observation of fine structure of $\mathrm{BN}$ and $\mathrm{ABN}$ modes in photoluminescence (PL) spectra of
PM. We demonstrated that $\mathrm{BN}$ and $\mathrm{ABN}$ modes consist of grouped supernarrow [full width at half maximum $(\mathrm{FWHM})=0.1 \rightarrow 0.4 \mathrm{~nm}]$ resonances with linewidth, spacing, and the total number depending on the angular mode number $n$ of the individual noninteracting microspheres. The observed structure reflects the complexity of photonic states distribution in coupled modes of PM and arises from lifting of the WGM degeneracy with respect to the azimuthal quantum number $m$. To make this observation requires a controllable orientation of the photonic molecule. We have used a microporous polymer structure that permits excitation and detection both on and off axis.

In order for the strong coupling phenomenon to be observed in PL spectra of the PM, the interacting spheres should be almost equal in size [7] and the quantum efficiency of the emitting species high enough to provide an efficient self-scattered field. In our experiments MF microspheres of 3 $\mu \mathrm{m}$ in diameter (Particle Size Standard, Microparticles $\mathrm{GmbH}$, Berlin) were coated with highly luminescent CdTe NCs utilizing the layer-by-layer deposition technique described elsewhere [12,13]. The high refractive index $\left(\mathrm{n}_{\mathrm{r}}\right.$ $=1.68$ ) and optical transparency of MF make it an ideal candidate for optical applications while CdTe NCs produce sizedependent emission which can be tuned in a wide spectral region. In this work a colloidal solution of CdTe NCs of 2.9 $\mathrm{nm}$ in size with a PL maximum at $563 \mathrm{~nm}$ and a PL quantum efficiency of $\sim 10 \%$ was used to coat the MF microspheres.

The small size of the MF spheres used was dictated by specific requirements for optimal excitation conditions, such as matching the laser wavelength to one of the WGM frequencies and achieving good correlation between the WGM and the laser linewidths [2,14]. The wide separation of WGM in spheres of this size allows us to avoid intricate band mixing in the PM. Moreover, the quality factor of the $3 \mu \mathrm{m}$ microspheres forming the $\mathrm{PM}\left(Q \sim 10^{3}\right)$ provides better mode coupling than would be in the case with larger microspheres [7]. We proceed from the assumption that a larger coupling parameter is of crucial importance in the interaction between electromagnetic fields of two spheres and necessary 


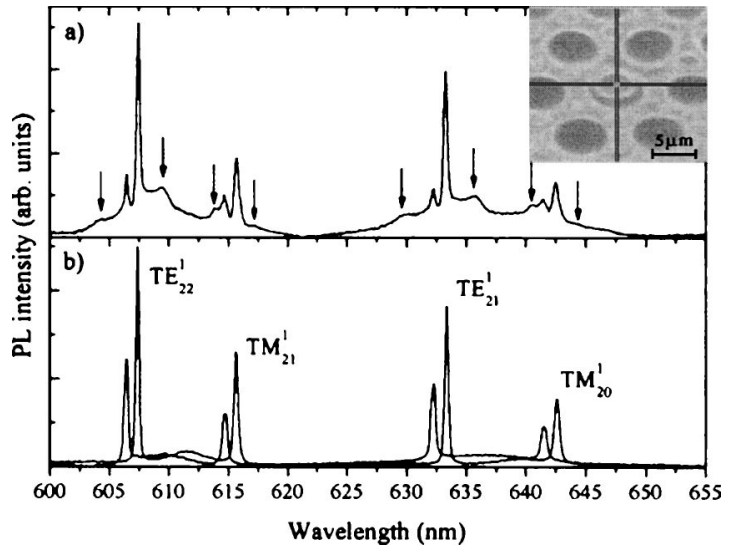

FIG. 1. (a) The PL spectrum of a PM accommodated in a microwell. Arrows indicate the coupled modes. (b) The PL spectra of noninteracting microspheres. Inset: microscope image of the PM in the microwell. The dark cross indicates the excitation position.

to experimentally study the fine spectral structure of coupled WGM.

Taking into account that the signal from the coupled microsphere modes is expected to be more pronounced when the incident light propagates parallel to the longitudinal axis of the PM [7], we employed a technique to control the alignment of the spheres constituting the PM. We utilized a polystyrene substrate containing a three-dimensionally-ordered array of pores of $\sim 5 \mu \mathrm{m}$ in size prepared through a thermocapillary convection [15]. The surface layer of the substrate contains an array of open microwells of 3-5 $\mu \mathrm{m}$. Only one pair of microspheres of $3 \mu \mathrm{m}$ size can be accommodated within each microwell, and for which the axis of the PM is close to the surface normal. In the experiments detailed here, $\mathrm{MF} / \mathrm{CdTe}$ microspheres were deposited on top of the porous substrate and then manipulated into position with a tapered optical fiber tip attached to a mechanical translation stage.

The PL spectra from the microspheres were recorded in a backscattering geometry using a micro-Raman system (Renishaw plc) as described elsewhere [13]. By recording the PL spectra of individual spheres placed on the top of the substrate and/or confined in a microwell, we can select spheres with the appropriate size. Because of the high PL quantum efficiency of the NCs, the WGM peaks in the PL spectra are superimposed on a background signal arising from part of the NC emission, which does not match any WGM of the microsphere $[13,14]$. This PL background has been subtracted in all the spectra presented in this paper to show the WGM structure more clearly. It is worth noting that the WGM structure in the PL spectra of single microspheres is found to be practically unaffected by the microporous substrate, except for a very small broadening of the WGM resonance lines. This can be understood by taking into account the difference in size between the microspheres and the microwell. The gap between the microwell walls and the microsphere can efficiently preserve the high $\mathrm{Q}$ value of the WGMs.

Figures 1(b) and 1(a) show the PL spectrum of a PM accommodated in a microwell and the spectra of individual microspheres. The observed spectral structure originates from the coupling of the electronic transitions in CdTe NCs and the photon states of MF microspheres [13,14], with PL peaks corresponding to the resonant frequencies of WGM with transverse electric (TE) and transverse magnetic (TM) polarizations. According to the Lorentz-Mie theory, the position and linewidth of the WGM can be uniquely determined by calculation of the scattering crosssection or solving equations for the denominators of the scattering coefficients $[2,16]$. Comparing results with the spectral positions of the WGM in experimental PL spectra we can identify the indices $n$ and, $l$ [Figs. 1(a) and 1(b)] for each mode and estimate the sizes of the spheres.

Pronounced doublet structure in the spectrum presented in Fig. 1(a), coinciding with the spectral positions of WGM of individual microspheres [Fig. 1(b)], is a result of the overlap of uncoupled modes of the two spheres. The shift in position of the $T E_{22}^{1}$ mode, which can be clearly seen in Figs. 1(a) and $1(b)$, is a result of a difference in the size of the two microspheres of only $25 \mathrm{~nm}$ as follows from our calculations. However, in contrast to the spectrum in Fig. 1(b), this doublet is accompanied by two extra relatively broad peaks [indicated by arrows in Fig. 1(a)], which are indicative of strong mode coupling in the PM. The appearance of these two satellites can be interpreted as a result of the formation of $\mathrm{BN}$ and $\mathrm{ABN}$ orbitals in the PM [5] with the $\mathrm{ABN}$ peak observed at lower wavelength than the $\mathrm{BN}$ one. In terms of cooperative scattering theory, [5,6] the observed satellites originate from the removal of the WGM degeneracy with respect to the $m$ index. The line shape of the satellite lines reflects the energy distribution among the coupled modes, because modes with different combinations of $m$ can contribute to the PL spectra. A recent theoretical study showed that the split modes from the PM are expected to be broader than the corresponding single-sphere modes [6], which is in agreement with our experimental results. The high PL efficiency of CdTe NCs and the coupling of electronic transitions of NCs to the resonances of the PM allows us to detect the $\mathrm{BN}$ and $\mathrm{ABN}$ branches in a wide spectral region from 525 to $725 \mathrm{~nm}$ corresponding to the full spectral range of the $Q D$ emission. We have found a decrease in splitting between $\mathrm{BN}$ and $\mathrm{ABN}$ branches value with increasing $n$, with TE modes splitting being always higher than that of TM modes. Remarkably, the theory predicts the same behavior [5].

Careful theoretical consideration of the internal electromagnetic fields associated with the WGM in the spectra of the PM pointed out that controllable alignment geometry of $\mathrm{PM}$ is crucial in order to observe the strong coupling between the spheres [7]. In all previous work on the BN-ABN splitting, the PM was excited and detected in the direction parallel to the PM axis (on axis) [7] or in the perpendicular configuration $[5,10,11]$. However it turns out that the interaction between modes of the same $n$ but different $m$ numbers can be more clearly revealed for off-axis (but not perpendicular) incidence.

One can see from the inset in Fig. 1(a) that excitation and detection in this case occured at the centre of the upper sphere forming the PM, i.e., along the PM axis. In order to reveal the $m \neq \pm 1$ components in the PL spectra of the PM we have investigated spectra at different excitation (and detection) positions across the upper sphere. 


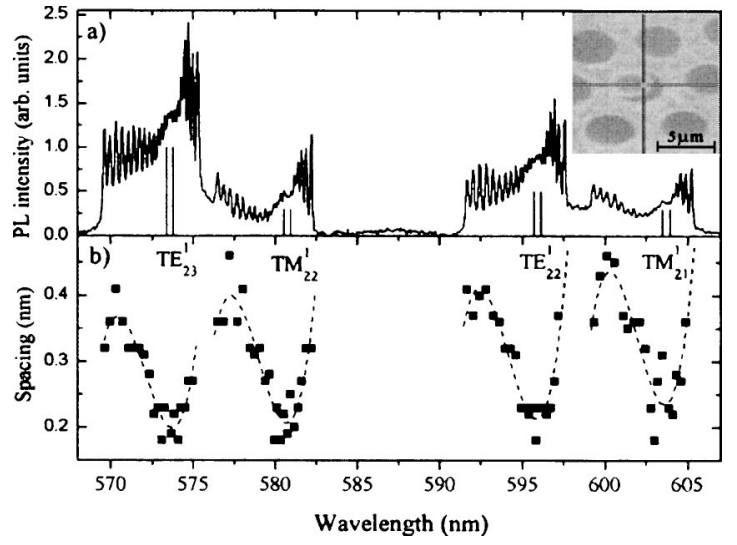

FIG. 2. (a) The PL spectra of the PM formed by two almost identical microspheres with off-axis excitation and detection. The vertical lines indicate the spectral position of the WGM detected in spectra of the individual spheres. (b) The spacing between neighboring peaks. The dashed lines are a guide to the eye. Inset: microscope image of the PM. The dark cross indicates the excitation position.

Figure 2(a) shows PL spectra of a PM formed by two almost identical microspheres (sample $B$ ) with sizes of $3.0168 \mu \mathrm{m}$ and $3.0189 \mu \mathrm{m}$ for which both excitation and signal collection occured at the right edge of the upper microsphere [inset, Fig. 2(a)]. One can clearly see a number of narrow peaks grouping on both sides arising from the TE and TM resonances of the individual spheres, which are the signature of the removal of the WGM degeneracy in the PM. The number of experimentally observed peaks increases with the angular mode number both for the TE and TM modes, and is very close to the $n$ value, although never in excess of $n$ [Fig. 2(a)]. It was found that the spacing between the $m$ resonances and their intensity decreases significantly when approaching the WGM resonance position of the individual spheres [Fig. 2(b)], which makes it difficult to distinguish between them at this point. We believe that this experimental limitation is the reason why the number of observed peaks is always no more than $n$, although at least an $(n+1)$ dependence is expected [5]. Also, we would like to stress the three major features which can be clearly seen in Figs. 2(a) and 2(b): (1) the WGM resonances of individual spheres correspond very well to the wavelength where the minimum peak separation occurs in the spectrum of the PM; (2) for all branches we have always observed an initial increase of the spacing within the region of first three peaks followed by a dip and then an increase in spacing again, and (3) the peak intensity in PL spectra of the PM decreases as it approaches the Mie resonances of the individual spheres. Gaining a better insight into the first two experimental findings, we have estimated the spacing between peaks with varying $m$ numbers, which differ by 1 (Fig. 3). First, we adopted the maximum term approximation of the single-mode tight-binding method [Eqs. (4.8a), (4.9), (C14), and (C15a) in Ref. [5]] in order to calculate the magnitude of the splitting between the $\mathrm{BN}$ and $\mathrm{ABN}$ modes $\Delta \mathrm{x}_{\mathrm{m}}$ for the $T E_{23}^{1}$ resonance in the range $0<m<10$ taking the size parameter $\mathrm{x}=16.5286$ and the microsphere radius $R=1.51 \mu \mathrm{m}$. The positions of the $m$ reso-

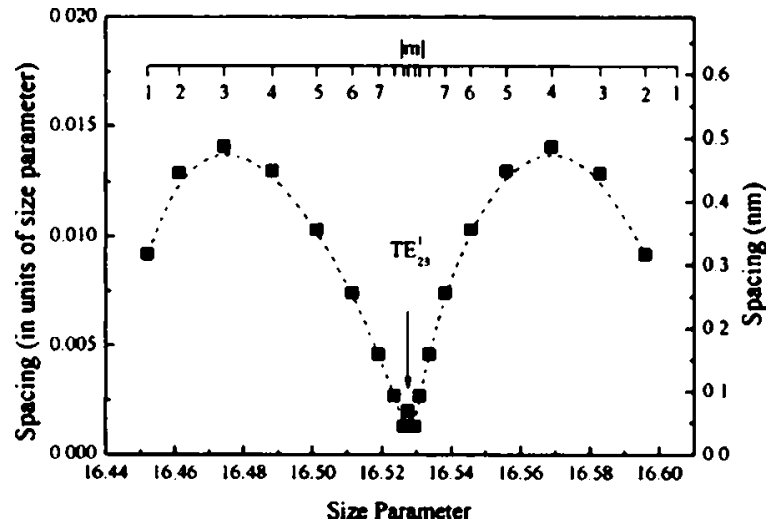

FIG. 3. Spacing between adjacent peaks estimated for different values of azimuthal quantum number $(m)$ from calculation of the splitting between bonding and antibonding modes. The dashed line is a guide to the eye.

nances were estimated as $x_{\mathrm{m}}=x \pm \Delta \mathrm{x}_{\mathrm{m}} / 2$ Eventually, the spacing between adjacent peaks was calculated as $\left(x_{\mathrm{m}+1}\right.$ $\left.-x_{\mathrm{m}}\right)$. Although the maximum term approximation overestimates the splitting, this approach provides a good qualitative guide for the analysis of a variety of phenomena observed in PM's [4,5]. In order to achieve qualitative comparison with experimental data the spacing was estimated in nanometers using the equation for the size parameter $(x=2 \pi R / \lambda)$.

We can see that the modeled data, presented in Fig. 3 displays behavior which is in good agreement with experimental data [Fig. 2(b)]. First, the spacing between $m$ resonances is smaller for larger $m$. Second, one can see an initial increase in the spacing between the first three peaks, although the distinctive discrepancy between the predicted and experimental behavior can be seen in the region of longer wavelength. For example, in the region between $\mathrm{TE}_{23}^{1}$ and $\mathrm{TM}_{22}^{1}$ resonances we did not observe any decrease in spacing magnitude [Figs. 2(a) and 2(b)]. Such apparent disagreement

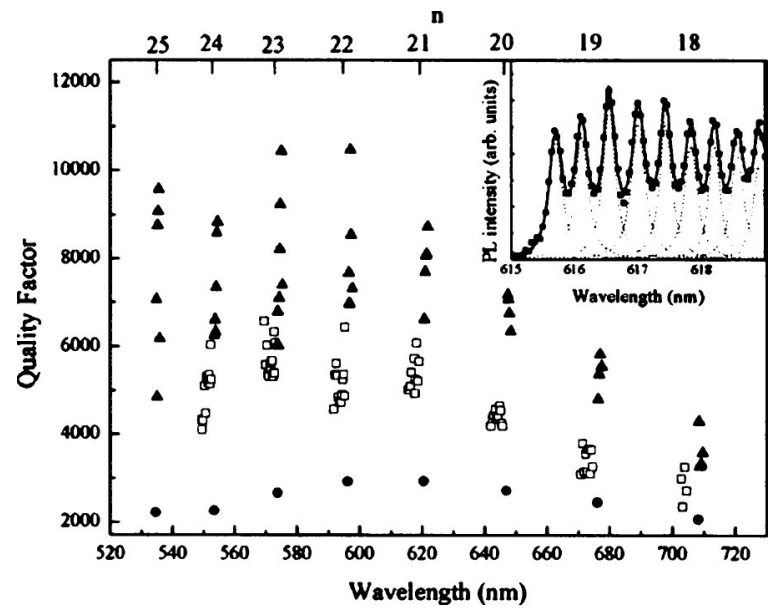

FIG. 4. Quality factor calculated for $m$ resonances forming bonding (squares) and antibonding (triangles) branches of PM. Quality factor estimated for one of individual spheres forming the PM (circles) is given for comparison. Inset: The deconvolution of the line shape of $m$ resonances belonging the $T E_{21}^{1}$ antibonding branch of a photonic molecule. 
with theory can be explained by the suppression of the low $m$ peaks due to enhanced interaction with the second order $T E_{19}^{2}$ resonances of the individual spheres, which is also responsible for the observed asymmetry of the BN-ABN splitting. However, the agreement between the magnitude of the spacing value between experiment [Fig. 2(b)] and theory (Fig. 3) is worthy of note. Finally, the position of the $\mathrm{TE}_{23}^{1}$ resonance of a single sphere closely matched the minimum in the spectral distribution of spacing between $m$ resonances (Fig. 3). Analysis of the internal energy of the PM (which can be obtained by integrating the energy density within the volume of two spheres [5]) reveals a pronounced decrease in intensity of $m$ resonances with increasing $m$, again in agreement with our data. This behavior (along with observation of smaller spacing for larger $m$ ) suggests that the interaction of modes decreases with increasing $m$ and can be explained by a corresponding decrease in the expansion coefficient for the incident wave electromagnetic field [5]. In order to explain the pronounced gaps observed in the spectral regions between TE and TM modes [Fig. 2(a)], we suggest a much weaker interaction between modes of different polarizations.

Our data clearly show that the BN and ABN modes of PM actually consist of a number of very narrow peaks, which are due to the presence of $m \neq \pm 1$ components. The total number of these $m$ resonances originating from a certain mode $n$ is governed by the degeneracy of the Mie resonances, which in the approach of the normal modes is $(n+1)$ [5]. In the case of on-axis or perpendicular orientation of the PM with respect to excitation, all these modes would merge into broad $\mathrm{BN}$ and $\mathrm{ABN}$ features and their fine structure would not be easily recognized [8]. The insert in Fig. 4 shows the deconvolution of the line shape of the $m$ resonances belonging to the $\mathrm{TE}_{21}^{1}$ $\mathrm{ABN}$ branch of PM using Lorentian functions. It turns out that $m$ resonances of the $\mathrm{BN}$ branch are always sharper than those of the ABN one, providing a higher $Q$ value for these modes (Fig. 4).

In summary, we have observed and explained the fine structure and the optical spectra of photonic molecules. The dependence of splitting parameters on the characteristics of noninteracting species suggests a manifold of applications, in particular in the field of quantum cryptography, quantum computing, and quantum information processing.

This work was supported by Science Foundation Ireland under Grant Nos. 02/IN.1/I47 and 00/PI.1/C077A.2, and by the Deutsche Forschungsgemeinschaft through the SPP "Photonic Crystals."
[1] K.J. Vahala, Nature (London) 424, 839 (2003).

[2] Optical Processes in Microcavities, edited by R.K. Chang and A.J. Campillo (World Scientific, Singapore, 1996).

[3] E. Lidorikis, M.M. Sigalas, E.N. Economou, and C.M. Soukoulis, Phys. Rev. Lett. 81, 1405 (1998).

[4] S. Arnold et al., J. Opt. Soc. Am. B 9, 819 (1992).

[5] M. Bayer et al., Phys. Rev. Lett. 81, 2582 (1998).

[6] K.A. Fuller, Appl. Opt. 30, 4716 (1991).

[7] T. Mukaiyama et al., Phys. Rev. Lett. 82, 4623 (1999).

[8] H. Miyazaki and Y. Jimba, Phys. Rev. B 62, 7976 (2000).
[9] S. Arnold et al., Opt. Lett. 19, 156 (1994).

[10] Y. Hara et al., Opt. Lett. 28, 2437 (2003).

[11] M.D. Barnes et al., Phys. Rev. Lett. 88, 015508 (2002).

[12] A.S. Susha et al., Colloids Surf., A 163, 39 (2000).

[13] Y.P. Rakovich et al., Semicond. Sci. Technol. 18, 914 (2003).

[14] Y.P. Rakovich et al., Appl. Phys. Lett. 83, 2539 (2003).

[15] M. Srinivasarao, D. Collings, A. Philips, and S. Patel, Science 292, 79 (2001).

[16] J.A. Stratton, Electromagnetic Theory (McGraw-Hill, New York, 1941). 